\title{
VAC_09 - Production and process control of a Meningococcal $W$ conjugate vaccine in a laboratory scale
}

Iaralice Medeiros de Souza ${ }^{1 *}$; Milton Neto da Silva ${ }^{1}$; Renata Chagas Bastos ${ }^{1}$; Denise da Silva Gomes Pereira $^{1}$; Elza Cristina Schott Figueira ${ }^{1}$; Ellen Jessouroun ${ }^{1}$; Maria de Lourdes Moura Leal ${ }^{1}$; Eliana BarretoBergter $^{2}$; Ivna Alana Freitas Brasileiro da Silveira ${ }^{1}$.

${ }^{1}$ Fiocruz/Bio-Manguinhos;

${ }^{2}$ UFRJ - Universidade Federal do Rio de Janeiro.

Introduction: Bio-Manguinhos was founded with the objective of meningococcal polysaccharide vaccines production. Since mid-2000s the Institute started to develop a Neisseria meningitidis serogroup C conjugate vaccine. Recent changes in meningococcal epidemiology have been reported and meningococcal serogroup W (MenW) became the third most prevalent serogroup isolated in Brazil in the last 10 years.

Objective: The aim of this study was to produce MenW conjugate bulks in lab scale, evaluate their immunological response and establish process controls.

Methodology: MenW strain 2467, from Adolfo Lutz Institute, was cultivated using Frantz medium in a 1501 bioreactor under stirring, $\mathrm{pH}$ and temperature control for $20 \mathrm{~h}$. After bacterial cells inactivation the supernatant was obtained by centrifuged, concentrated and precipitated with Cetavlon and Celite as filtration assistant. Elution and extraction was done with ammonium chloride, calcium chloride and ethanol to obtain purified polysaccharide. The conjugates production using reductive amination methodology started with NaIO4 polysaccharide oxidation. Tetanus toxoid was activated using hydrazine chloride to introduce hydrazide groups in residues of aspartic and glutamic acids. Conjugation studies indicated that the reaction was best conducted with concentration of polysaccharide twice as high as protein, at room temperature, and $\mathrm{pH}$ approximately 6.0. Bulks process control was done by physicochemical analysis such as PS and protein quantification, HPLC-SEC, capillary electrophoresis (CE) and ${ }^{1} \mathrm{H}$ nuclear magnetic resonance (NMR). A scaled up bulk was formulated and inoculated intramuscularly in mice using different doses $(0.1,0.5,1.0$ and $10 \mathrm{ug} / \mathrm{dose})$ in order to obtain immunized serum for ELISA and serum bactericidal antibody assay (SBA).

Results: Bioreactor growth showed that cells reached stationary phase in $6 \mathrm{~h}$ with continuous glucose consumption and polysaccharide production. Purified polysaccharide was obtained in accordance with WHO requirements and contained $2.38 \%$ nucleic acid, $0.69 \%$ protein, and $58.93 \%$ sialic acid. The scaled-up bulk produced showed conjugation ratio of around 0.5 , value observed in some multivalent meningococcal vaccines. HPLC-SEC analysis showed peaks with higher molecular weight than the ones observed in intermediary molecules in all batches, suggesting the existence of a polysaccharide:protein linkage. ${ }^{1} \mathrm{H}$ NMR analysis showed assignments related to hydrogens from galactose, sialic acid and protein. Quantification of free components in bulks by CE showed quantities of these molecules below the ones required by WHO. ELISA revealed high IgG titers in all doses studied however significant differences were observed among them $(\mathrm{p}=0.005)$. SBA titers were from $2^{8}-2^{10}$ demonstrating the antibodies functionality with no significant differences among studied doses $(\mathrm{p}=0.2387)$.

Conclusion: These results determined the best conditions to produce MenW conjugate vaccine and showed the efficacy of the obtained conjugate bulk in induce a good immune response in mice. Further experiments will need to be done to scale up the conjugation reaction and then allow the use of this conjugate in clinical trials.

Keywords: Meningococcal vaccine; Conjugate vaccine; Serogroup W meningococcal 